\title{
Cohesive strength of iron ore granules
}

\author{
Rafael Jaimes Contreras ${ }^{1}$, Nicolas Berger ${ }^{2,3}$, Edouard Izard $^{4, *}$, Jean-François Douce ${ }^{4}$, Alexey Koltsov ${ }^{4}$, \\ Jean-Yves Delenne ${ }^{5}$, Emilien Azema ${ }^{3}$, Saeid Nezamabadi ${ }^{3}$, Frédéric van Loo $^{1}$, Roland Pellenq ${ }^{2}$ and Farhang \\ Radjai $^{2,3}$ \\ ${ }^{1}$ Centre for research in Metallurgy, 4000 Liège, Belgium \\ ${ }^{2}$ UMI 3466 CNRS-MIT, Massachusetts Institute of Technology, MA 02139, USA \\ ${ }^{3}$ Université de Montpellier, CNRS, LMGC, 34095 Montpellier, France \\ ${ }^{4}$ ArcelorMittal R\&D Maizières, Voie Romaine, F-57283, Maizières-Lès-Metz, France \\ ${ }^{5}$ SupAgro, INRA, 34060 Montpellier, France
}

\begin{abstract}
We present an experimental and numerical investigation of the mechanical strength of crude iron ore (Hematite) granules in which capillary bonds between primary particles are the source of internal cohesion. The strength is measured by subjecting the granules to vertical compression between two plates. We show that the behavior of the granules is ductile with a welldefined plastic threshold which increases with the amount of water. It is found that the compressive strength scales with capillary cohesion with a pre-factor that is nearly independent of size polydispersity for the investigated range of parameters but increases with friction coefficient between primary particles. This weak dependence may be attributed to the class of fine particles which, due to their large number, behaves as a cohesive matrix that controls the strength of the granule.
\end{abstract}

\section{Introduction}

Since thirty years, iron ores dedicated to sinter plant show a downward trend of their quality (finer particles, broader size distributions, lower grades and larger fluctuations of properties [1]). This trend leads to a significant increase of the input rate of very fine particles in the sinter plants causing productivity (and sometimes sinter quality) drops. A direct and intuitive countermeasure to this evolution is to enhance the input materials in the so-called granulation step, where fine particles are agglomerated into coarse granules allowing for adequate sinter bed permeability on the strand. The granulation process is carried out in continuous drum granulators where water is used as binder [2]. Besides iron ores, this process involves multiple components such as return fines, solid fuel (coke breeze or anthracite), by-products and fluxes (limestone, olivine and dolomite [3]).

The present study aims at developing a better understanding of the physical mechanisms involved in the agglomeration process in view of quantifying and predicting the mechanical strength of the granules. To isolate those mechanisms, we study a laboratory model of granules. These model granules are produced in a laboratory-scale batch drum granulator with iron ore controlled in size and nature. Rather than impact tests $([4,5])$, we perform compression tests $[6,7]$ in order to measure the strength of the granules as a function of granulation parameters. In parallel, simulations of compression tests are performed by means of the Discrete Element Method [8,9] (DEM) coupled to a model of capillary bonding. Our goal is to establish a clear relationship between grain-scale cohesion and texture, on one hand, and the granule macroscopic strength, on the other hand.

In the following, we first describe the procedures used to prepare the laboratory model of granules and the compression experimental tests. Then, discrete element simulations using a cohesive force are briefly introduced and simulations of compression tests are presented.

\section{Experimental methods and results}

The raw material used in the granulation experiments is a commercial iron ore identified as hematite ore from which a sieve size fraction of diameters below $0.25 \mathrm{~mm}$ was extracted. The chemical analysis of the raw material is presented in Table 1. The primary particles are mixed to achieve a homogeneous mixture (Figure 1A) that was used throughout this work.

Table 1. Chemical analysis of the raw material

\begin{tabular}{|c|c|}
\hline Constituents & $\mathbf{\%}$ \\
\hline Fe total & 64 \\
\hline $\mathrm{CaO}$ & 0.06 \\
\hline $\mathrm{SiO} 2$ & 6.46 \\
\hline Loss of ignition & 1.03 \\
\hline
\end{tabular}


Let us first present the experimental procedure used to create laboratory model granules. A stainless steel batch granulation drum of $8.5 \mathrm{~cm}$ in diameter and 4.25 $\mathrm{cm}$ long, without scraper or lifter bar, was used to create granules which were then subjected to compression test. Tap water is injected in the drum and acts as binder to agglomerate the particles. A needle with an internal diameter of $0.4 \mathrm{~mm}$ is used to spray the binder with the same flow rate and injection time for all tests. The homogeneous mixture of hematite ore is introduced into the drum, as shown in Figure 1B. The drum starts to rotate up to the constant value of $17 \mathrm{rpm}$. Then, a predefined amount of water is poured by means of the needle. The drum rotates during three minutes while the granules are being formed. Finally, the granules are collected and their size distribution is measured by standard manual sieving.

Compression tests are performed on the granules between two parallel plates as shown in Figure 2. A force gauge allows for the measurement of the compressive force in combination with a motorised motion system which stops or reverses at a set load. The load increments and displacements of the plates are collected thanks to the EmperorTM software. As the load increases, the granule deforms and fails. The axial force increases, peaks and drops at failure to a lower value as shown in Figure 3. The deformation of the granule beyond the peak is plastic with a nearly constant force level $F^{*}$ (up to fluctuations around the mean). $F^{*}$ has nearly half the peak value. A sensitivity analysis on the platen speed was conducted, showing that a velocity of $1 \mathrm{~mm} / \mathrm{min}$ is sufficiently low to reproduce peak force results. The statistical errors are evaluated by repeating the test. Figure 4 shows the range of the values of the peak force for two different compression speeds. Ten granules of identical diameters (with an error of $11.48 \%$ ), collected from drum granulator experiments were used for each series of tests. We see that the variability is smaller at lower speed. The peak force depends also on the moisture content as observed in Figure 5. The peak force increases with the water content up to $10.1 \%$ in volume, then levels off at a value of $5 \mathrm{~N}$ for higher water content. At this level, the liquid is in the funicular state [10].

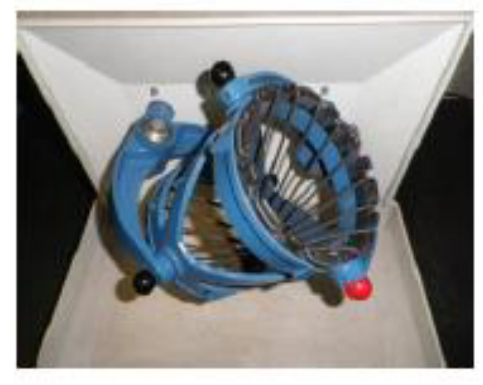

A

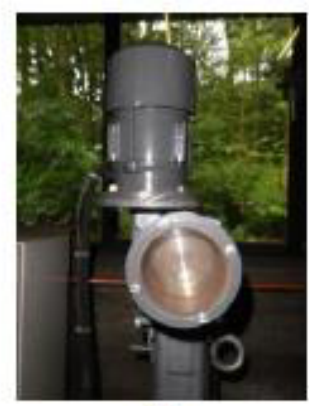

B
Fig. 1. Experimental set-up for iron ore granulation: picture (A) shows the turbula shaker-mixer used for homogenisation procedure while picture (B) illustrates the drum granulator used to create granules.

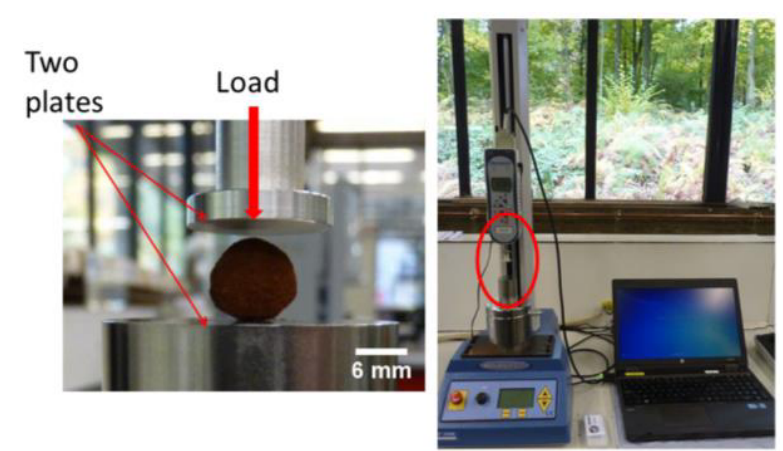

Fig. 2. The setup used for compression test on iron ore granules. The granule is compressed between two platens and the time series of the compressive force with the downward displacement of the top plate is recorded.

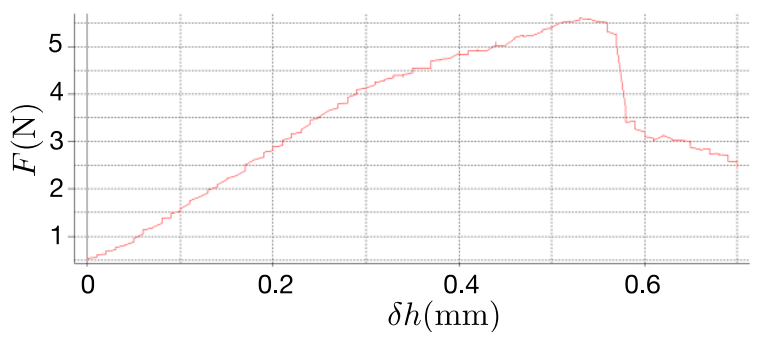

Fig. 3. Typical force-displacement curve during diametrical compression of a granule for water content $w=11.1 \%$.

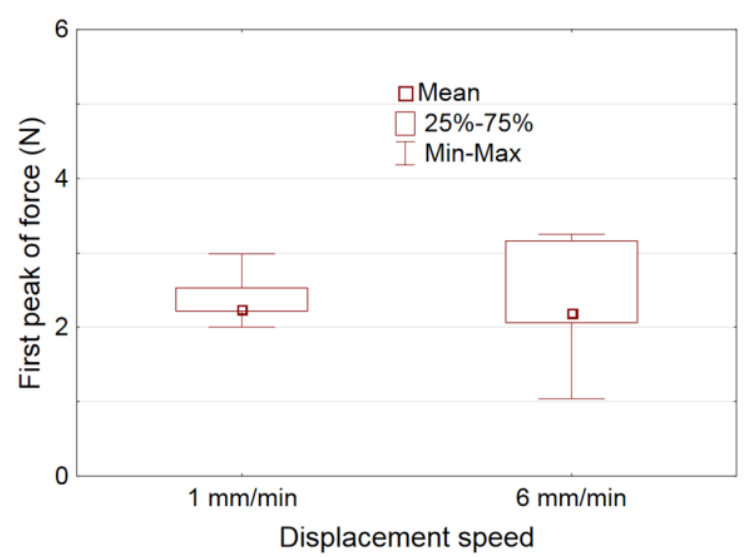

Fig. 4. Impact of load speed on the first peak force measured in the compression experimental test.

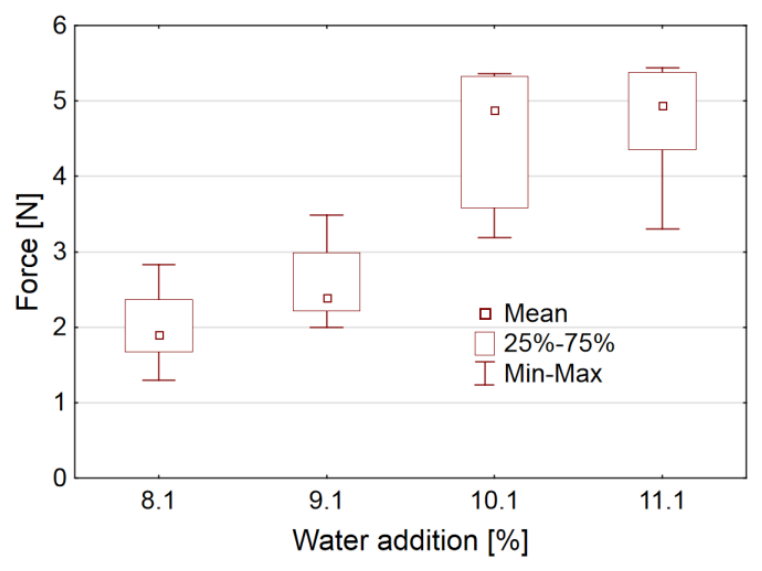

Fig. 5. First peak force as a function of water content in the hematite granule. The force increases with water content. The granule size varies slightly with the amount of water. 


\section{Discrete Element simulations}

The compressive strength of iron ore granules, as measured from experiments, reflects the capillary cohesion of primary particles. For smallest amounts of water used in experiments, it may be assumed that the water is distributed as binary liquid bridges between particles $[11,12]$. For the simulations, we also assume that the capillary forces act only at the contact points between particles and the contact angle is zero. The capillary force at the contact point between two particles of diameters $d_{i}$ and $d_{j}$ is given by $f_{c}=\pi \gamma_{s} \sqrt{d_{i} d_{j}}$ where $\gamma_{s}$ is the liquid-vapor surface tension. These forces induce a cohesive strength proportional to $2 \pi \gamma_{s} /\langle d\rangle$.

The strength $\sigma_{a}$ of the granule can be defined from value of the peak force $F$ divided by the average section $\pi R^{2}$ of the granule, e.g. $\sigma_{a}=F /\left(\pi R^{2}\right)$.

We expect that $\sigma_{a}$ is proportional to the cohesive strength. Hence, we can define the following dimensionless strength:

$$
\eta_{c}=\frac{\sigma_{a}}{2 \pi \gamma_{s} /\langle d\rangle}
$$

This ratio may depend on the size distribution of primary particles, friction coefficient between particles and the connectivity of the contact network. From the experimental value of $\sigma_{a}$ in the low water content limit, the average size of the primary particles $(\langle d\rangle \approx 6.8 \mu \mathrm{m})$ and using $F^{*} \approx 1 \mathrm{~N}$, we get $\eta_{c} \approx 0.2$ for our experiments.

In order to investigate the effects of various parameters on the value of $\eta_{c}$, we used DEM simulations with varying size polydispersity and different values of the friction coefficient between particles. For the numerical modeling of granules, we used the Contact Dynamics (CD) method [8,9] with spherical primary particles together with the expression of the capillary force $f_{c}$. The size span of the primary particles in experiments being quite broad, we are computationally limited by the number of particles. The number of particles required to account correctly for size polydispersity increases rapidly with the ratio $\lambda=$ $d_{\max } / d_{\min }$ between the maximum diameter $d_{\max }$ and minimum diameter $d_{\min }$. It is necessary to satisfy both the statistical representativeness of all sizes in their number (especially for the largest particles) and in their volume (especially for the classes of finest particles) [13]. For this reason, we consider four size classes with diameters $d_{i}(i \in[1,2,3,4])$ and 8 values of $\lambda$ ranging from 1 (monodisperse) to 4.5 with increments of 0.5 . For the shape of the size distribution, we used a uniform distribution by volume fractions of particles such that the particle volumes in all classes are equal. This choice was motivated by the fact that a uniform volume distribution of particles leads to a sufficient number of fine particles to allow the effect of size polydispersity to be manifest on the compressive strength of the granule. The granules are composed of nearly 5000 primary particles.

To build the granules, we first create a dense assembly by raining frictionless particles into a box and allowing them to relax to equilibrium. Then, we extract a spherical volume from this assembly. This method allows us to control the characteristics of the granules such as their size, compactness, connectivity and homogeneity. In the absence of friction, the granules are rather dense with the packing fraction increasing from 0.59 to 0.68 as $\lambda$ varies from 1 to 4.5 . As in experiments, the granules are subjected to diametrical compression between two walls. Figure 6 displays a granule at three instants of compression with force chains between primary particles.
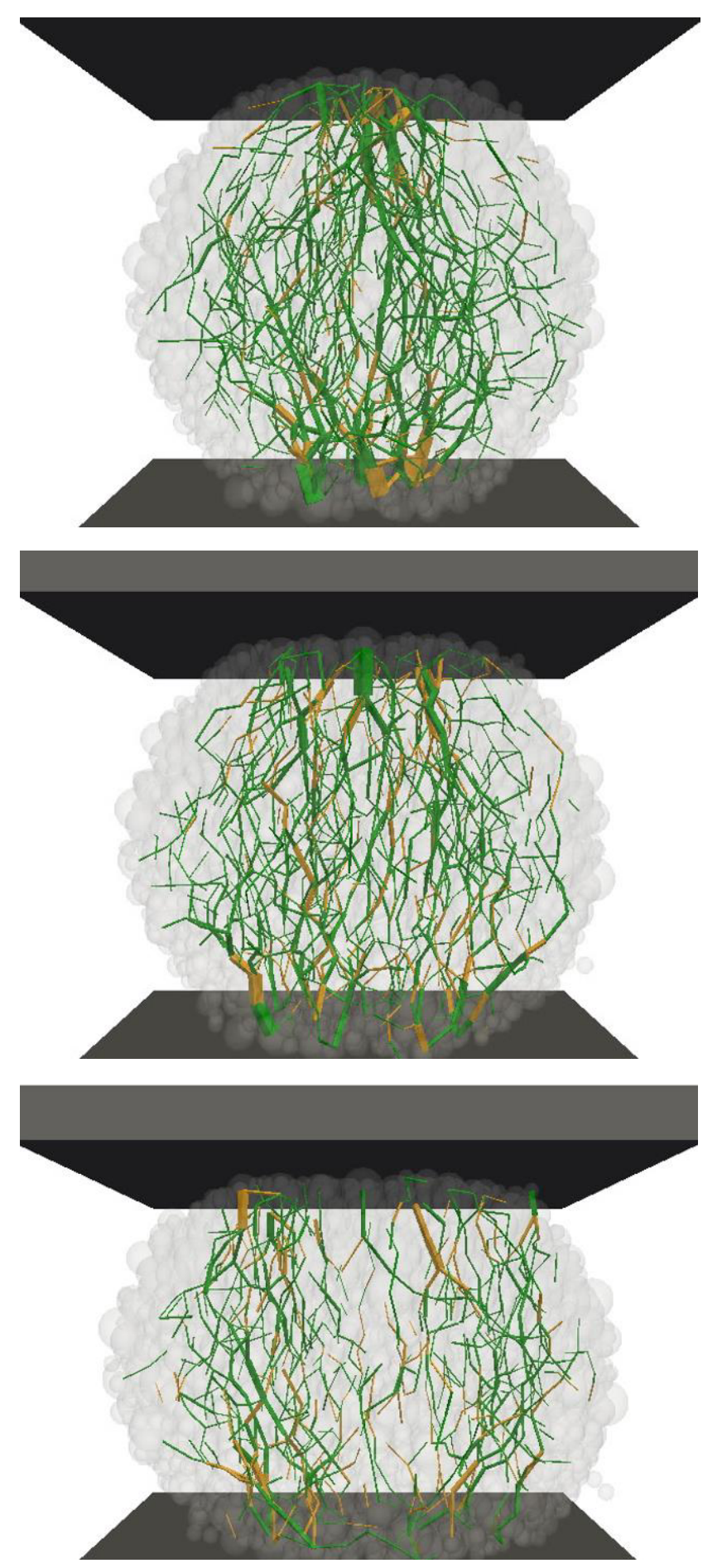

Fig. 6. A granule at three instants of diametral compression. Line thicknesses are proportional to normal forces between primary particles. The compressive forces are in green (black for black and with print) color whereas tensile forces are in orange color (gray for black and with print). 
The stress-strain curves for all granules with friction coefficient $\mu_{s}=0.7$ between primary particles are plotted in Figure 7. As a result of force fluctuations due to finite size effects, the first stress peak cannot be easily identified; thus, we consider the mean value $\mathrm{F}^{\wedge *}$ of the force in the plastic regime. Table 2 presents the values of the mean calculated force and dimensionless strength parameter $\eta_{c}$. We see that $\eta_{c} \approx 0.2$ independently of $\lambda$ and in excellent agreement with experiments.

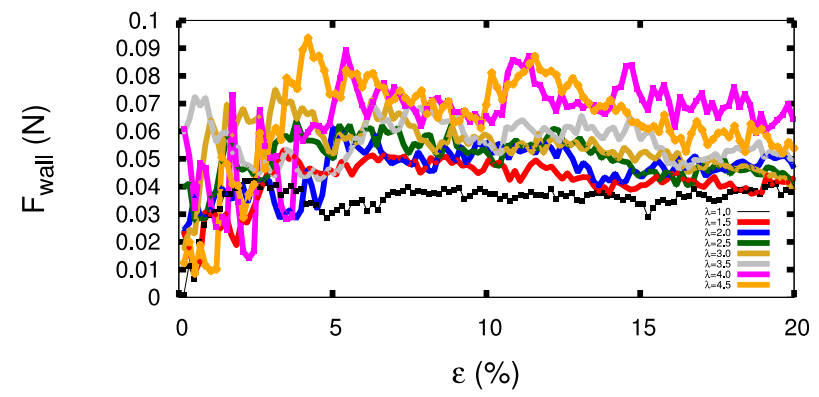

Fig. 7. A Compressive force on the top wall as a function of vertical deformation $\varepsilon=\delta h / 2 R$ for all value of size ratio $\lambda$.

Table 2. The plastic threshold $F^{*}$, initial radius $R$, mean diameter $\langle d\rangle$ of the primary particles and the value of dimensionless strength parameter $\eta_{c}$ for different granules with different size ratios $\lambda$.

\begin{tabular}{|l|l|l|l|l|}
\hline \multicolumn{1}{|c|}{$\lambda$} & \multicolumn{1}{c|}{$F^{*}(\mathrm{~N})$} & $R(\mathrm{~mm})$ & $\langle d\rangle(\mathrm{mm})$ & $\eta_{c}$ \\
\hline 1 & 0.035 & 16.5 & 2.00 & 0.20 \\
\hline 1.5 & 0.048 & 19.8 & 2.34 & 0.22 \\
\hline 2 & 0.048 & 21.9 & 2.46 & 0.19 \\
\hline 2.5 & 0.055 & 22.8 & 2.49 & 0.21 \\
\hline 3 & 0.057 & 23.3 & 2.48 & 0.21 \\
\hline 3.5 & 0.056 & 23.6 & 2.45 & 0.19 \\
\hline 4 & 0.065 & 24.3 & 2.41 & 0.21 \\
\hline 4.5 & 0.072 & 24.5 & 2.40 & 0.23 \\
\hline
\end{tabular}

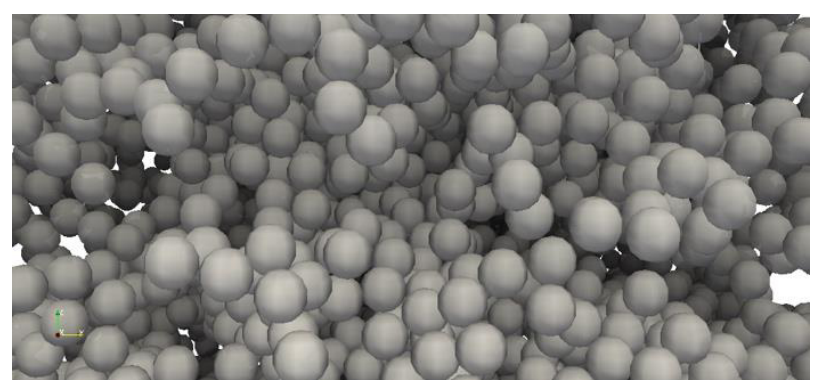

Figure 8: A zoom on the organization of the class of finest particles in a granule with $\lambda=4$.

We checked that the same granules with $\mu_{s}=0.4$ lead to the same strength. But for $\mu_{s}=0.1$, the value of $\eta_{c}$ declines by a factor 2 . This shows that the friction coefficient can influence the granule strength in the range of 0 to 0.4 . Microstructural analysis (not shown in this paper) indicates that, for a uniform distribution of particle volume fractions, the cohesion is mainly controlled by the class of finest primary particles and hence it weakly depends on the size distribution although it crucially depends on the average size. This class behaves as a cohesive matrix with a large interface with all size classes. Figure 8 shows the class of fine particles in the simulations with $\lambda=4$, where we see how they form a connected matrix filling the whole volume of the granule. Hence, as far as the class of fine particles is large in number, including larger primary particles in the granule does not affect much the overall cohesion of the granules. This is intuitively the case when nuclei of large size are added to the agglomeration process.

\section{Conclusion}

In this paper, we presented an experimental and numerical investigation of the strength of agglomerates composed of primary spherical particles as a function of water content (in experiments) and increasing size polydispersity (in simulations). The relevant parameter in the analysis of the strength is the plastic threshold normalized by the cohesive capillary stress induced by liquid bonds. We found nearly the same value both from experiments and DEM simulations using the Contact Dynamics method. The independence of normalized strength with respect to size polydispersity can be understood from the fact that the cohesion of the granule is mainly controlled by the class of fine particles. This class, because of its large number of particles and volume fraction, behave as a homogeneous matrix with a large interface with particles in all size classes.

\section{References}

1. R. Remus, M. A. Aguado Monsonet, S. Roudier, \& L. Delgado Sancho, JRC reference report. Best Available Techniques (BAT) Reference Document for Iron and Steel Production (2013)

2. J. Litster, B. Ennis. The science and engineering of granulation processes (Vol. 15). (Springer Science \& Business Media, 2013)

3. D. F. Ball, Agglomeration of Irons Ores, (New York : American Elsevier Pub. Co., 1973)

4. S. Antonyuk, M. Khanal, J. Tomas, S. Heinrich \& L. Mörl, Chemical Engineering and Processing: Process Intensification, 45(10), 838-856 (2006)

5. S. Antonyuk, J. Tomas, S. Heinrich, L. Mörl, Chem. Eng. Sci., 60(14), 4031-4044 (2005)

6. S. Antonyuk, S. Heinrich, J. Tomas, N. G. Deen, M. S. van Buijtenen, J. A. M. Kuipers, Granular Matter, 12(1), 15-47 (2010)

7. A. Russell, P. Müller, J. Tomas, Chem. Eng. Sci., 114, 70-84 (2014).

8. M. Jean, J. J. Moreau, Proceedings of Contact Mechanics International Symposium, pp. 31-48 (1992)

9. F. Radjai, V. Richefeu, Mech. Mat. 41, 715-728 (2009)

10. J.-Y. Delenne, V. Richefeu, F. Radjai, J. Fluid Mech., 762 R5 (2014)

11. T. C. Halsey, A. J. Levine. Phys. Rev. Lett., 80(14), 3141 (1998)

12. A. Gladkyy, R. Schwarze, Granular Matter, 16(6), 911-920 (2014)

13. C. Voivret, F. Radjaï, J.-Y. Delenne, M. S. El Youssoufi, Phys. Rev. E 76, 021301 (2007) 